Eduardo R. Butelman · S. Stevens NeguS

John W. Lewis · James H. Woods

\title{
Clocinnamox antagonism of opioid suppression of schedule-controlled responding in rhesus monkeys
}

Received: 27 July 1995 / Final version: 19 October 1995

\begin{abstract}
The antagonist effects of clocinnamox were evaluated against opioid agonists, acting at $\mu, \kappa$ and $\partial$-receptors, in rhesus monkeys $(n=3-4)$ responding under a fixed-ratio 30 (FR 30) schedule for food delivery. Clocinnamox $(0.032-0.1 \mathrm{mg} / \mathrm{kg})$ dose-dependently antagonized fentanyl $(0.001-0.32 \mathrm{mg} / \mathrm{kg})$ after either a $3-\mathrm{h}$ or 1-day pretreatment; there was substantial recovery of agonist potency by 1 week after clocinnamox. Etonitazene $(0.0001-0.01 \mathrm{mg} / \mathrm{kg})$ was also antagonized by clocinnamox $(0.1 \mathrm{mg} / \mathrm{kg})$, but to a lesser extent than fentanyl. The smaller extent of antagonism was not due to the appearance of non $\mu$-opioid response-decreasing effects of etonitazene, since the competitive antagonist quadazocine $(0.1 \mathrm{mg} / \mathrm{kg})$ shifted the etonitazene dose-effect curve in the presence of clocinnamox $(0.1 \mathrm{mg} / \mathrm{kg})$. Clocinnamox $(0.1-0.32 \mathrm{mg} / \mathrm{kg})$ did not antagonize the ratesuppressing effects of the $\partial$-agonist BW373U86 (0.0.01$1.0 \mathrm{mg} / \mathrm{kg})$ or the $\mathrm{K}$-agonist U69,593 $(0.001-0.032$ $\mathrm{mg} / \mathrm{kg}$ ). These results are consistent with previous in vivo and in vitro evidence that characterized clocinnamox as an insurmountable antagonist, with selectivity for $\mu-$ over $\kappa$ - and $\delta$-receptors.
\end{abstract}

Animals used in these studies were maintained in accordance with the University Committees on the Use and Care of Animals, University of Michigan, and Guidelines of the Committee on the Care and Use of Laboratory Animals of the Institute of Laboratory Animal Resources, National Health Council (Department of Health, Education and Welfare, Publication No. (NIH) 85-23, revised 1983). Support for this research was provided by USPHS grant DA 00254.

E. R. Butelman ( ) S. S. Negus ${ }^{1}$. J. H. Woods

Department of Pharmacology, University of Michigan,

1301 MSRB III, Ann Arbor, MI 48109, USA

J. H. Woods

Department of Psychology, University of Michigan, Ann Arbor, MI 48109, USA

J. W. Lewis

Department of Chemistry, University of Bristol, Bristol, UK

Present address:

1. ADARC, Department of Psychiatry, Harvard Medical School,

115 Mill St, Belmont, MA 02178, USA
Key words Clocinnamox $\cdot$ Fentanyl $\cdot$ Irreversible antagonists - Operant behavior · Rhesus monkeys

\section{Introduction}

Irreversible opioid antagonists such as $\beta$-funaltrexamine ( $\beta$-FNA) have been used as a way to study both the selectivity and the efficacy of different agonists in a variety of experimental conditions, in vivo (e.g., Zimmerman et al. 1987; Adams et al. 1990; Mjanger and Yaksh 1991). The cinnamoylmorphinone, clocinnamox, has recently been characterized in tests of antinociception in mice and monkeys (Comer et al. 1992; Burke et al. 1994; Zernig et al. 1994), and was described as an irreversible opioid antagonist with relative selectivity for $\mu$ - over $\kappa$ - and $\partial$-opioid receptors. An advantage of clocinnamox relative to $\beta$-FNA in behavioral studies is that the former does not have an initial, probably $\kappa$-receptor mediated (e.g., Ward et al. 1982) agonist effect which limits behavioral testing soon (i.e., $<24$ h) after $\beta$-FNA administration. Burke et al. (1994) reported a correspondence between the antagonist effects of clocinnamox against the antinociceptive effects of morphine in mice and an observed decrease in the number of $\mu$-receptor binding sites in mouse brain. Thus, clocinnamox (IP, $3.2 \mathrm{mg} / \mathrm{kg}$ ) produced an almost total suppression in $\left[{ }^{3} \mathrm{H}\right] \mathrm{DAMGO}$ ([D-Ala ${ }^{2}, N-M e-P h e^{4}$, glycol$\left.{ }^{5}\right]$-enkephalin) binding sites in mouse brain, peaking $1 \mathrm{~h}$ after administration, and the number of sites substantially recovered by 8 days after administration. At this dose, clocinnamox caused a large right and downward shift in the morphine dose-effect curve in thermal antinociception, and clocinnamox did not have an antinociceptive effect by itself. The time course of the suppression of morphine's antinociceptive effects mirrored well the decrease in $\left[{ }^{3} \mathrm{H}\right]$ DAMGO binding sites (Burke et al. 1994). More recent in vitro and ex vivo experiments in mice confirmed that clocinnamox exhibited wash-resistant binding at $\mu$-, but not $\kappa-$ or $\partial$-, receptors, thus supporting the in vivo selectivity of its 
antagonist effects (Zernig et al. 1994, 1995; Broadbear et al, manuscript in preparation).

In the present studies, we examined whether clocinnamox presented an opioid antagonist profile in a non-antinociceptive behavioral assay in rhesus monkeys, i.e., suppression of schedule-controlled food-reinforced responding. This procedure is a simple means to study the pharmacology of $\mu$ - (e.g., etonitazene and fentanyl), $\kappa-($ e.g., U69,593), and $\partial-$ (e.g., BW373U86) opioid agonists in vivo in rhesus monkeys (Negus et al. 1993, 1994), and is especially helpful in evaluating clocinnamox's $\mu$-over $\partial$-selectivity, given that the only nonpeptide $\partial$-agonist characterized to date, BW373U86, is inactive as a thermal analgesic in rhesus monkeys, but does suppress food-reinforced responding through a $\partial$ receptor mechanism (see Negus et al. 1994; Butelman et al. 1995b).

\section{Materials and methods}

\section{Subjects}

The subjects were seven adult male and female rhesus monkeys, weighing between 7 and $12 \mathrm{~kg}$ during the course of the study. They were housed individually with free access to water and controlled amounts of Purina monkey chow, supplemented by fresh fruit at least twice per week.

\section{Apparatus}

The apparatus and other experimental details have been described previously (Negus et al. 1993). During experimental sessions, one of the subjects was placed in a standard primate restraint chair, which was then placed inside a sound-attenuating behavioral cubicle equipped with a panel containing two levers and stimulus lights above the levers. The remaining subjects were tested in their home cages, which were also equipped with a panel containing two levers and stimulus lights. Experimental panels had a food receptacle mounted centrally, just below the panels themselves. Externally mounted pellet dispensers delivered $300 \mathrm{mg}$ banana flavored pellets (P.J. Noyes Co., Lancaster, N.H.). All programming and data collection were carried out with IBM PCjr computers.

\section{Experimental sessions}

Sessions were carried out 5-6 days/week; during training days, sessions were composed of five consecutive 15-min cycles. Each cycle was composed of an initial 10-min timeout period during which responses on any lever had no programmed consequence. This was followed by a 5 -min response period, during which stimuls lights were illuminated and 30 lever presses resulted in pellet delivery (FR30). Subjects could obtain a maximum of ten pellets/cycle.

\section{Test sessions}

During test sessions, agonist doses were administered at the beginning of each cycle, using a cumulative dosing procedure, increasing by $1 / 2 \log$ unit steps. Dosing continued until responding was eliminated in each subject, or after the appearance of untoward ef fects, such as preconvulsant behavior in the presence of BW373U86. Dose-effect curves were determined for etonitazene, fentanyl, BW373U86 and U69,593. These dose-effect curves were redetermined $3 \mathrm{~h}$ (fentanyl only), 1 day and 1-2 weeks after pre- treatment with single clocinnamox doses $(0.032-0.32 \mathrm{mg} / \mathrm{kg}$, $n=3-4)$. In another experiment, the etonitazene dose-effect curve was also redetermined 30 min after pretreatment with quadazocine $(0.1 \mathrm{mg} / \mathrm{kg})$, either alone or 1 day after clocinnamox pretreatment $(0.1 \mathrm{mg} / \mathrm{kg})$.

Data analysis

Rates of responding (responses per second) were collected for each subject individually, and were expressed as a percentage of the mean control rate, determined from the previous training day. Individual $\mathrm{ED}_{50}$ values were calculated by linear regression from points above and below the $50 \%$ level. Mean $\mathrm{ED}_{50}$ and $95 \%$ confidence limits (95\% CL) were calculated after log transformation of individual $\mathrm{ED}_{50}$ values.

Drugs

Quadazocine methanesulfonate (Sterling Winthrop, Rensselaer, N.Y.), fentanyl hydrochloride (NIDA, Rockville, Md.), etonitazene hydrochloride (NIDA, Rockville, Md.), BW373U86 [( $( \pm)-4-\left(R^{*}\right)$ $\left(\left(2 S^{*} 5 R^{*}\right)\right.$-4-allyl-2,5,-dimethyl-1-piperazinyl)-3-hydroxybenzyl)$N, N$-diethylbenzamide dihydrochloride] (Burroughs Wellcome, Reasearch Triangle Park, N.C.), U69,593 (Upjohn Co., Kalamazoo, Mich.) and clocinnamox mesylate (Dr. John Lewis, Bristol University, UK) were all dissolved in sterile water and injected SC in the thigh or the back, typically at volumes of $0.1 \mathrm{ml} / \mathrm{kg}$ body weight. All drug doses were expressed as salt weights.

\section{Results}

\section{Control performance}

Subjects were easily trained on the schedule, and typically yielded stable rates of responding for all the cycles in training sessions. Control rates of responding ranged from two to four responses/s for all the monkeys studied.

\section{Agonist dose-effect curves}

All of the opioid agonists suppressed dose-dependently and completely the rates of food-reinforced responding in all the monkeys tested. At doses that completely suppressed responding, no obvious signs of intoxication were present. The $\mathrm{ED}_{50}$ values for suppression of responding by each of the agonists are presented in Table 1.

\section{Antagonist effects of clocinnamox}

Clocinnamox alone did not affect rates of responding on the day it was administered (not shown). When clocinnamox $(0.032 \mathrm{mg} / \mathrm{kg})$ was administered before fentanyl, a rightward shift in the fentanyl dose-effect curve, approximately $1 \log$ unit in magnitude, was observed $24 \mathrm{~h}$ after pretreatment $\left(\mathrm{ED}_{50}=0.019 \mathrm{mg} / \mathrm{kg}\right)$. The fentanyl dose-effect curve had returned to baseline by 1 week after clocinnamox $(0.032 \mathrm{mg} / \mathrm{kg}$, Fig. 1). When a higher clocinnamox dose $(0.1 \mathrm{mg} / \mathrm{kg})$ was administered before 
Table $1 \mathrm{ED}_{50}$ values for opioid agonists alone and 1 day after pretreatment with clocinnamox $(0.1 \mathrm{mg} / \mathrm{kg})$

\begin{tabular}{|c|c|c|c|c|c|}
\hline & \multicolumn{2}{|c|}{ Agonist alone } & \multicolumn{2}{|c|}{$0.1 \mathrm{mg} / \mathrm{kg}$ Clocinnamox } & \multirow[t]{2}{*}{ D.R.b } \\
\hline & $\mathrm{ED}_{50^{\mathrm{a}}}^{\mathrm{a}}$ & $( \pm 95 \% \mathrm{CL})$ & $\mathrm{ED}_{50}$ & $( \pm 95 \% \mathrm{CL})$ & \\
\hline Etonitazene & 0.0005 & $0.0004-0.0006$ & 0.003 & $0.0007-0.01 *$ & 6 \\
\hline Fentanyl & 0.002 & $0.001-0.006$ & 0.06 & $0.01-0.3^{*}$ & 30 \\
\hline BW373U86 & 0.09 & $0.009-0.8$ & 0.1 & $0.003-3.6$ & 1.1 \\
\hline U-69,593 & 0.004 & $0.001-0.01$ & 0.009 & $0.003-0.03$ & 2.3 \\
\hline
\end{tabular}

$\mathrm{aED}_{50}:$ dose required to reduce response rates to $50 \%$ of control

b D.R.: dose ratio, i.e. [ $\mathrm{ED}_{50}$ clocinnamox/ED ${ }_{50}$ agonist alone]

* Significant shift in dose-effect curve, as defined by lack of overlap in $95 \%$ confidence limits $(95 \%$ CL)
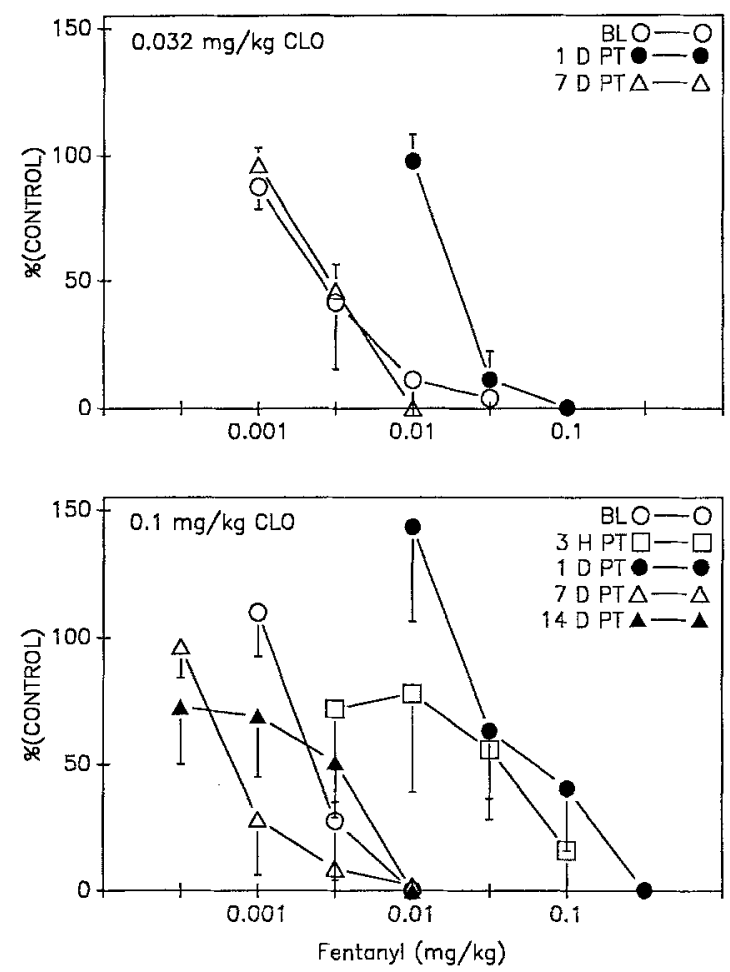

Fig. 1 Time course of the antagonist effects of clocinnamox (CLO, 0.032 and $0.1 \mathrm{mg} / \mathrm{kg}$, upper and lower panels, respectively) pretreatment against fentanyl. Upper panel: fentanyl dose-effect curves were redetermined 1 and 7 days $(D)$ after a single clocinnamox $(C L O, 0.032 \mathrm{mg} / \mathrm{kg})$ pretreatment. Lower panel: fentanyl dose-effect curves were redetermined $3 \mathrm{~h}(3 \mathrm{H})$, and 1,7 , and 14 days after clocinnamox $(0.1 \mathrm{mg} / \mathrm{kg}$. Data in the lower panel were obtained in two separate experiments, one in which the $3-\mathrm{h}$ time point alone was tested, another in which the remaining time points were tested. Abscissae: dose of fentanyl (mg/kg); ordinates: rate of responding expressed as a percent of control, \pm SEM

fentanyl, a larger shift of the fentanyl dose-effect curve, approximately $1.5 \mathrm{log}$ units in magnitude, was observed both 3 and $24 \mathrm{~h}$ later (the two dose-effect curves were carried out after two separate clocinnamox administrations; see Table 1). The fentanyl dose-effect curve also returned to approximate baseline levels within 1 week of 0.1 clocinnamox pretreatment (Fig. 1).

Clocinnamox $(0.1 \mathrm{mg} / \mathrm{kg})$ also shifted the etonitazene dose-effect curve 1 day after administration (see Fig. 2 and Table 1). The magnitude of the shift was $0.75 \mathrm{log}$ units, approximately, and complete recovery was ob-
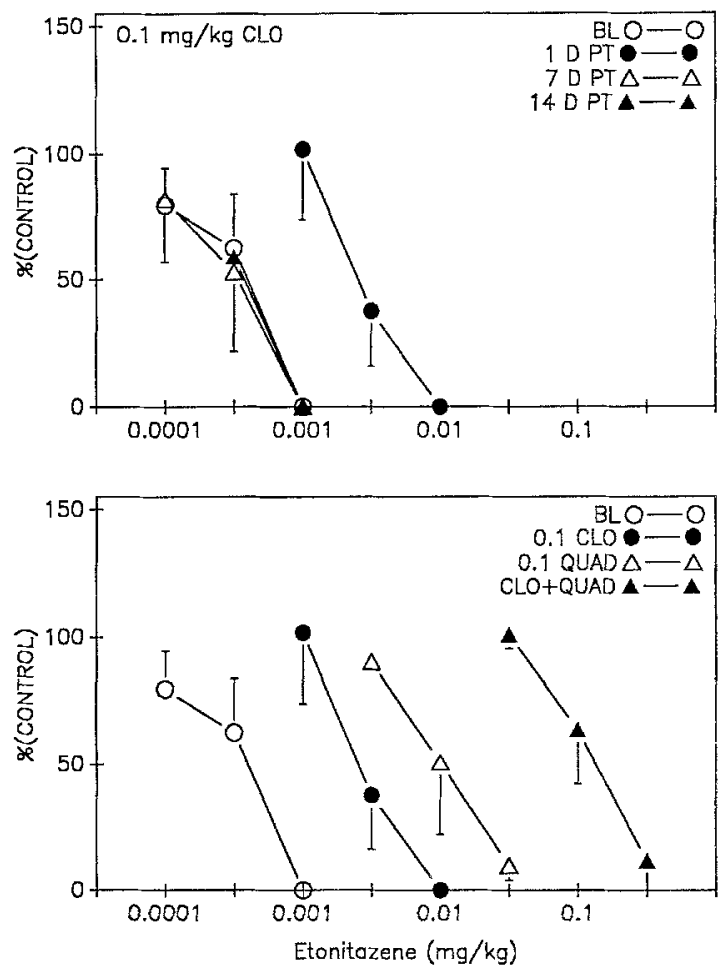

Fig. 2 Upper panel: time course of the antagonist effects of clocinnamox $(0.1 \mathrm{mg} / \mathrm{kg})$ against etonitazene. Lower panel: effect of quadazocine ( $Q U A D, 0.1 \mathrm{mg} / \mathrm{kg}$ ) pretreatment, $30 \mathrm{~min}$ before etonitazene, tested in the presence or the absence of clocinnamox $(0.1 \mathrm{mg} / \mathrm{kg}$ ). Etonitazene baseline $(B L)$ and $0.1 \mathrm{mg} / \mathrm{kg}$ clocinnamox pretreatment are repeated in the two panels. All other details as in Fig. 1

served by 1 week after clocinnamox. In a separate experiment, the competitive opioid antagonist quadazocine $(0.1 \mathrm{mg} / \mathrm{kg})$ was administered $30 \mathrm{~min}$ before etonitazene, either $24 \mathrm{~h}$ after clocinnamox $(0.1 \mathrm{mg} / \mathrm{kg})$, or in its absence. Quadazocine $(0.1 \mathrm{mg} / \mathrm{kg})$ alone caused a significant rightward shift, approximately $1.5 \mathrm{log}$ units in magnitude, in the etonitazene dose-effect curve $\left(\mathrm{ED}_{50}=\right.$ $0.01 \mathrm{mg} / \mathrm{kg}$; Fig. 2). In the presence of clocinnamox, quadazocine $(0.1 \mathrm{mg} / \mathrm{kg})$ caused a similar shift, also approximately $1.5 \log$ units in magnitude, of the etonitazene dose-effect curve ( $E D_{50}=0.14 \mathrm{mg} / \mathrm{kg}$; Fig. 2). (0.1 $\mathrm{mg} / \mathrm{kg}$ ) did not antagonize the rate-decreasing effect of BW373U86, and only caused a small and non-significant shift in the U69,593 dose-effect curve (Table 1). A larger clocinnamox pretreatment dose $(0.32 \mathrm{mg} / \mathrm{kg})$ was also 
studied in combination with U-69,593 and BW373U86. This clocinnamox dose caused no further shift in the rate-decreasing effects of U69,593 or BW373U86, $24 \mathrm{~h}$ after administration (data not shown).

\section{Discussion}

Clocinnamox has previously been described as an irreversible opioid antagonist in tests of antinociception in mice and rhesus monkeys (Comer et al. 1992; Burke et al. 1994; Zernig et al. 1994). The present findings extend the characterization of its antagonist effects in a different assay, suppression of operant, food-reinforced responding, which has been previously used to characterize $\mu$-, $\kappa-$ and $\partial$-opioid agonists and antagonists (e.g., Negus et al. 1993; 1994). In the present study, clocinnamox $(0.032-0.32 \mathrm{mg} / \mathrm{kg})$, in common with other opioid antagonists (see Negus et al. 1993, 1994), did not cause by itself a suppression in operant responding, but dose-dependently and robustly antagonized the effects of the $\mu$ agonists etonitazene and fentanyl. The peak antagonist actions of clocinnamox $(0.1 \mathrm{mg} / \mathrm{kg})$ against etonitazene and fentanyl were observed up to 1 day after administration, and there was substantial or complete recovery by 1 week. A similar time course was observed with thermal antinociception in rhesus monkeys, in which clocinnamox $(0.1 \mathrm{mg} / \mathrm{kg})$ antagonism of alfentanil's actions had substantially diminished by 1 week after pretreatment (Zernig et al. 1994). The $\mu$-antagonist effects of clocinnamox $(0.1 \mathrm{mg} / \mathrm{kg})$ were surmountable in the present test of response rate suppression, but insurmountable in a test of antinociception in rhesus monkeys (i.e., $50^{\circ} \mathrm{C}$ warm water tail withdrawal; Zernig et al. 1994). This difference could suggest the presence of a larger receptor reserve for response rate suppression than for antinociception, or that a lower level of receptor occupancy is required for the former endpoint than for the latter. Consistent with this, the opioid agonist doses required for response rate suppression tend to be smaller than those required for antinociception (e.g., present study; Walker et al. 1993; France et al. 1994).

Conversely, clocinnamox did not significantly antagonize the rate-decreasing effects of a $\mathrm{K}-(\mathrm{U}-69,593)$ and a $\partial$ - (BW373U86) agonist, even at the highest dose that could be administered due to clocinnamox solubility $(0.32 \mathrm{mg} / \mathrm{kg})$. It has previously been shown that the agonist effects of U-69,593 and BW373U86 in this procedure were mediated by $\kappa$ - and $\partial$-receptors, respectively, as determined in quantitative studies with the competitive antagonists, quadazocine and naltrindole (Negus et al. 1993, 1994). Thus clocinnamox's lack of antagonism of these two agonists in the procedure, shows that clocinnamox exhibits considerable selectivity for $\mu$ - over $\kappa$ and $\partial$-receptors in this procedure. The selectivity of clocinnamox for $\mu$-over $\kappa$-receptors in monkeys has previously been reported in the warm water tail withdrawal test of antinociception in rhesus monkeys (Zernig et al. 1994; Butelman et al. 1995a)). The selectivity of clo- cinnamox for $\mu$ - over $\partial$-receptors, however, could not be evaluated in the same procedure, because the prototypic non-peptide $\partial$-agonist, BW373U86 is inactive in the warm water tail withdawal assay (Negus et al. 1994). Thus the present data are, to our knowledge, the first to indicate clocinnamox's $\mu$-versus $\partial$-selectivity in a primate species. In binding studies, clocinnamox displayed approximately 30 -fold $\mu$-over $\kappa$-selectivity, and 6 -fold $\mu$ - over $\partial$-selectivity. Furthermore, only binding at $\mu$-, but not $\kappa^{-}$or $\partial$-, opioid sites was highly resistant to repeated washing (Zernig et al. 1995). This profile results in clocinnamox having considerable selectivity in vivo for $\mu$-receptors.

An interesting aspect of the $\mu$-antagonist effects of clocinnamox $(0.1 \mathrm{mg} / \mathrm{kg}, 24 \mathrm{~h}$ after administration $)$ in the present studies is that it apparently produced a larger shift of fentanyl's dose-effect curve compared to etonitazene's dose-effect curve. It has previously been suggested that varying agonist efficacy results in differential sensitivity to irreversible antagonism. For example, the $\mu$-selective irreversible antagonist, $\beta$-FNA, produced larger shifts in the antinociceptive dose-effect curves of agonists thought to have low efficacy, relative to agonists thought to have high efficacy. This trend was observed in rodents in a variety of assays (tail flick, hot plate, abdominal constriction) following either central or systemic administration (Zimmerman et al. 1987; Adams et al. 1990; Mjanger and Yaksh 1991). The present data therefore suggest that fentanyl has lower efficacy than etonitazene in this procedure. Etonitazene has previously been described as having higher efficacy than the fentanyl congener, alfentanil, in thermal antinociception in rhesus monkeys (Walker et al. 1995). Relevant to this issue, quadazocine $(0.1 \mathrm{mg} / \mathrm{kg})$ pretreatment caused a further shift in the etonitazene dose-effect curve in the presence of clocinnamox, of similar magnitude than observed in the absence of clocinnamox. This quadazocine pretreatment dose $(0.1 \mathrm{mg} / \mathrm{kg})$ would not antagonize the effects of $\partial$ - or $\kappa$-agonists in this procedure (see Negus et al. 1994). Therefore, the extent of the clocinnamox-induced shift in etonitazene's dose-effect curve was not limited by the appearance of non $\mu$-opioid receptor-mediated effects. An alternative explanation for clocinnamox's differential shift of the etonitazene and fentanyl dose-effect curves is the possibility that these compounds had differential affinity for putative $\mu$-receptor subtypes (e.g., Pasternak 1993). This explanation cannot be evaluated at present, due to a lack of information on $\mu$-subtype pharmacology in primates.

The present data show that clocinnamox acts as a relatively long-lasting, $\mu$-selective antagonist in rhesus monkeys in a non-antinociceptive endpoint, and that it is devoid of an initial agonist effect. These findings extend previous data obtained in the procedure with the competitive antagonists quadazocine and naltrindole (Negus et al. 1993, 1994). Thus clocinnamox, which shows insurmountable antagonist effects in tests of antinociception in primates (Zernig et al. 1993), can be studied in this simple operant procedure, which is sensitive to a variety 
of opioids that may be inactive in other behavioral endpoints, including tests of antinociception.

Acknowledgements The expert technical assistance of Ms. Lisa McMahan is gratefully acknowledged.

\section{References}

Adams JU, Paronis CA, Holtzman SG (1990) Assessment of relative intrinsic activity of mu-opioid analgesics in vivo by using beta-funaltrexamine. J Pharmacol Exp Ther 255: 1027-1032

Burke T, Woods JH, Lewis JW, Medzihradsky F (1994) Irreversible opioid anatagonist effects of clocinnamox on opioid analgesia and mu receptor binding in mice. J Pharmacol Exp Ther 271: $715-721$

Butelman ER, France CP, Woods JH (1995a) Agonist and antagonist effects of dynorphin $\mathrm{A}-(1-13)$ in a thermal antinociception assay in rhesus monkeys. J Pharmacol Exp Ther 275: 374-380

Butelman ER, Negus SS, Gatch MB, Chang KJ, Woods JH (1995b) BW373U86, a d-opioid receptor agonist, reverses bradykinin-induced thermal allodynia in rhesus monkeys. Eur $\mathrm{J}$ Pharmacol 277: 285-287

Comer SD, Burke TF, Lewis JW, Woods JH (1992) Clocinnamox: a novel, systematically-active irreversible opioid antagonist. $\mathbf{J}$ Pharmacol Exp Ther 262: 1051-1056

France CP, Medzihradsky F, Woods JH (1994) Comparison of $\kappa$ opioids in rhesus monkeys: behavioral effects and receptor binding affinities. J Pharmacol Exp Ther 268: 47-58
Mjanger E, Yaksh T (1991) Characteristics of dose-dependent antagonism by beta-funaltrexamine of the antinociceptive effects of intrathecal mu agonists. J Pharmacol Exp Ther 258: $544-550$

Negus SS, Burke TF, Medzihadsky F, Woods JH (1993) Effects of opioid agonists selective for mu, kappa and delta opioid receptors on schedule-controlled responding in rhesus monkeys: antagonism by quadazocine. J Pharmacol Exp Ther 267: 896-903

Negus SS, Butelman ER, Chang KJ, DeCosta BR, Winger G, Woods JH (1994) Behavioral effects of the systemically active delta opioid agonist BW373U86 in rhesus monkeys. J Pharmacol Exp Ther 270: 1025-1034

Pasternak GW (1993) Pharmacological mechanisms of opioid analgesics. Clin Neuropharmacol 16:1-18

Walker EA, Butelman ER, DeCosta BR, Woods JH (1993) Opioid thermal antinociception in rhesus monkeys: receptor mechanisms and temperature dependency. J Pharmacol Exp Ther 267: $280-286$

Walker EA, Zernig G, Woods JH (1995) Buprenorphine antagonism of mu opioids in the rhesus monkey tail withdrawal procedure. J Pharmacol Exp Ther 273: 1345-1352

Zernig G, Butelman ER, Lewis J, Woods JH (1994) In vivo determination of mu opioid receptor turnover in rhesus monkeys after irreversible blockade with clocinnamox. J Pharmacol Exp Ther 269: 57-65

Zernig G, Burke T, Lewis JW, Woods JH (1995) Clocinnamox blocks only mu receptors irreversibly: binding evidence. Regul Pept (in press)

Zimmerman D, Leander DJ, Reel J, Hynes MD (1987) Use of beta-funaltrexamine to determine mu opioid receptor involvement in the analgesic activity of various opioid ligands. J Pharmacol Exp Ther 241: 374-378 